\title{
Boost public support for food systems innovation
}

\author{
Publicly funded technological innovations, strategic policy implementation and private sector upscaling have \\ facilitated greater demand and lower costs for certain foods in the past. Can lessons be learned for transitioning \\ towards healthy, sustainable diets?
}

\section{Lindsay M. Jaacks}

W e have not seen improvements in diets of the magnitude seen in other areas of public health, such as clean water, clean air, smoking and road traffic injuries. The US government has recommended the consumption of fruits and vegetables since $1916^{1}$ - and yet in 1976-1980 only 9\% of US adults met the 5-a-day guidelines for fruits and vegetables ${ }^{2}$; by 2015 , that number was still just $9-12 \%$ (ref. $^{3}$ ). How can a transition to healthy and sustainable diets be achieved with the rapidity our current global food crisis demands? Lessons could be learned from examining historical shifts in food consumption.

In this issue of Nature Food, Moberg et. al. unpack major consumption shifts in three animal-source foods - farmed tilapia, milk and chicken - to glean information about how we might proactively replicate shifts of a similar magnitude for other foods ${ }^{4}$. Technological innovations that lowered the cost of these foods and increased their availability were critical for scaling up, whilst other factors, such as cultural affinity, were not necessary for widespread adoption. Government subsidies enabled these innovations, for example, through improved tilapia and chicken breeding technologies leading to increased production efficiency - thus, lowering costs to consumers.

Despite this important role of public spending on research and development (R\&D), public investment in food systems innovations in high-income countries has been falling in real terms since the global financial crisis of $2008^{5}$; the COVID19 pandemic will likely squeeze public spending on R\&D in this sector even further. Simultaneously, private investment in food systems R\&D has increased substantially ${ }^{6}$ (Fig. 1). As Moberg et al. find in studying the history of these three commodities, public-private partnerships are key to accomplishing systems-level change ${ }^{4}$. However, relying entirely on the private sector to transform food systems is unlikely to achieve societal goals of public health,

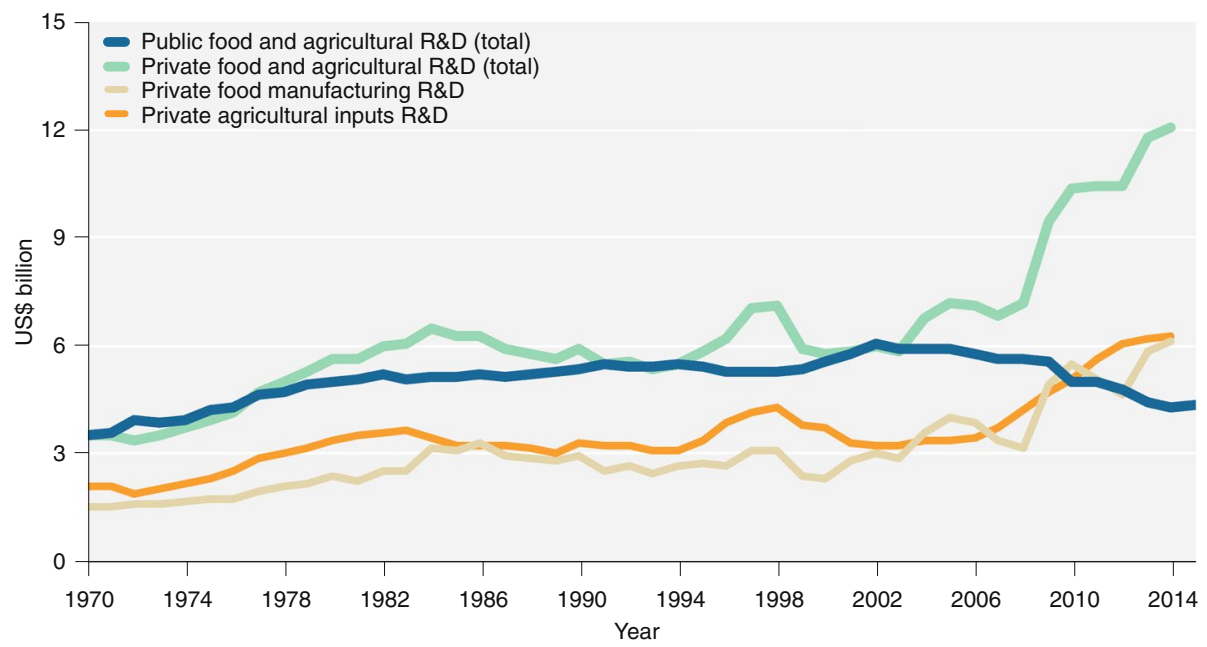

Fig. 1 | Investment in food systems research and development in the United States, 1970-2015. Values are in inflation-adjusted 2013 US dollars. R\&D, research and development. Credit: Reproduced with permission from ref. ${ }^{6}$, USDA Economic Research Service.

sustainability and equity. The authors point out that governments play an important role in regulating and endorsing certain foods, which can push or pull industry and consumers in the desired direction. One example discussed was the inclusion of milk in school lunch programmes and national dietary guidelines, which normalized consumption of this commodity from an early age.

Unplanned and unregulated food transitions can have unintended consequences - both negative externalities and co-benefits. The nutrition transition has contributed not only to the obesity epidemic and premature mortality from non-communicable diseases, but also to increased environmental footprints ${ }^{7}$. Data produced by the research community on these unintended consequences must reach policy-makers to inform the complex trade-offs associated with emerging technologies; policy-makers need to be proactive - rather than reflexive — in planning the scaling up of these technologies ${ }^{8}$. These so-called sociotechnical innovation bundles ${ }^{9}$ require careful designing with input from not just natural scientists, but also social sciences ${ }^{10}$.

To date, much public policy research on reducing the consumption of unhealthy food commodities has drawn from the tobacco playbook. Yet Moberg et al. find that many of these policies, such as meat taxes, front-of-pack labels, and raising awareness about the links between food and the environment, are unlikely to drive change of the scale required to meet international targets for health and sustainability ${ }^{4}$. The absence of these policies in historical transitions does not necessarily mean that they will not be relevant to future transitions. But, the authors' analysis of processes that shaped large-scale changes in the past suggests that these single-driver solutions are unlikely to have the transformative impact sought.

We have less than nine years to meet the Sustainable Development Goals and one critical question remains - how quickly can such transitions occur? 
The authors study 70 years of data, finding that technological innovations preceded increases in consumption by a decade or more ${ }^{4}$. Another recent evaluation of food systems transitions similarly found that even the most attractive innovations have a long pathway to impact $^{11}$. This is where 'transformation accelerators' come in - for example, near-universal uptake of high-yielding seed varieties was successful in Asia, but adoption of these technologies was limited in sub-Saharan Africa ${ }^{8}$. Contextual factors in Asia, including public investment in infrastructure (for example, irrigation, transportation and communications) and public pricing and procurement systems enabled this transition ${ }^{8}$. As Moberg et al. point out ${ }^{4}$, future studies of new technology or commodity adoption in separate contexts will improve understanding of complex transition pathways - and how to accelerate them.
Moberg and colleagues highlight that formulaic attempts to replicate past sequences of marketing, technology and state investment will not necessarily result in the desired transitions ${ }^{4}-\mathrm{a}$ conclusion that suggests there are several pathways to major, national dietary shifts. Thus, we must ensure that public investments in $\mathrm{R} \& \mathrm{D}$, infrastructure, endorsement and regulation drive innovation that simultaneously shifts populations towards healthier diets, reduces negative externalities on the environment and improves the quality of jobs in the agri-food sector. Whilst there is no single technology or silver bullet solution for the challenges facing food systems, there is hope that calls for contemporary diet and food-system reform are possible - and will be answered.

\section{Lindsay M. Jaacks (D) $₫$}

Global Academy of Agriculture and Food Security,
The University of Edinburgh, Midlothian, UK.

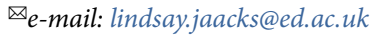

Published online: 15 April 2021

https://doi.org/10.1038/s43016-021-00239-3

References

1. Davis, C. \& Saltos, E. in America's Eating Habits: Changes and Consequences (ed. Frazão, E.) Ch. 2 (USDA, 1999).

2. Patterson, B. H., Block, G., Rosenberger, W. F., Pee, D. \& Kahle, L. L. Am. J. Public Health 80, 1443-1449 (1990).

3. Lee-Kwan, S. H., Moore, L. V., Blanck, H. M., Harris, D. M. \& Galuska, D. Morb. Mortal. Wkly Rep. 66, 1241-1247 (2017).

4. Moberg, E. et al. Nat. Food https://doi.org/10.1038/s43016-02100261-5 (2021).

5. Heisey, P. W. \& Fuglie, K. O. Agricultural Research Investment and Policy Reform in High-income Countries (USDA, 2018).

6. Agricultural Research Funding in the Public and Private Sectors (USDA Economic Research Service, 2019); http://go.nature. $\mathrm{com} / 304 \mathrm{fzNT}$

7. Willett, W. et al. Lancet 393, 447-492 (2019).

8. Herrero, M. et al. Nat. Food 1, 266-272 (2020).

9. Barrett, C. B. et al. Nat. Sustain. 3, 974-976 (2020).

10. Leach, M. et al. World Dev. 134, 105024 (2020)

11. Herrero, M. et al. Lancet Planet. Health 5, e50-e62 (2021).

Competing interests

The author declares no competing interests. 